\title{
Ungewisse Zeitrechnung
}

\section{Vorgeschichten und analeptisches Erzählen in Goethes Roman Wilhelm Meisters Wanderjahre}

\section{Cornelia Zumbusch}

Zusammenfassung Die fragmentierte und scheinbar kontingente Struktur von Goethes Roman Wilhelm Meisters Wanderjahre folgt, so soll hier gezeigt werden, nicht zuletzt auch einem besonderen Zeitregime. Der Roman erprobt vor allem in seiner um Wilhelm und Lenardo gruppierten Handlung Formen des analeptischen Erzählens, das die Ereignisse nicht chronologisch-linear, sondern in wiederholten Rückgriffen und Zeitschleifen entwickelt. Dieses Erzählen von und in Vorgeschichten folgt einem Schema, das Goethe in den homerischen Epen vorgeprägt sieht, das aber auch mit Zeitreflexionen aus seinen geologischen Studien korrespondiert.

\section{Uncertain Regime of Time}

Prehistories and analeptical Telling in Goethe's Wilhelm Meisters Wanderjahre

\begin{abstract}
The fragmented and apparently contingent structure of Goethe's novel Wilhelm Meisters Wanderjahre introduces, as will be shown here, a specific time regime. The novel unfolds the stories of its main characters Wilhelm and Lenardo in an analeptical fashion developing its events not in their chronological order, but in time loops and regressions. This narrative of and in >Vorgeschichten < (prehistories or backstories) harks back to Goethe's reading of Homeric epic and also corresponds to temporal reflections voiced in his geological studies.
\end{abstract}

\footnotetext{
C. Zumbusch $(\varangle)$

Fakultät für Geisteswissenschaften, Fachbereich Sprache, Literatur, Medien I, Institut für Germanistik, Universität Hamburg, Überseering 35, Postfach \#15, 22297 Hamburg, Deutschland E-Mail: cornelia.zumbusch@uni-hamburg.de
} 
»[I]ch mag kaum glauben, daß etwas gewesen sei was nicht noch da ist «. ${ }^{1}$ Diese Idee einer Persistenz des Alten im Neuen, die Vorstellung also, dass alles Vergangene irgendwo noch anzutreffen sei, bezeichnet einen neuralgischen Punkt der Wanderjahre. Der Satz fällt in einem Gespräch Wilhelms mit dem sogenannten Oheim, bei dem Wilhelm zu Beginn der Wanderjahre Station macht. Dieser Oheim bewohnt ein gut gesichertes und mit hohen Mauern umgebenes »Riesenschloß«, das er nicht nur zu einem hochproduktiven Agrarbetrieb, sondern auch zu einem Archivraum ausgebaut hat. Skeptisch gegenüber allen Bildern mit rein mythologischen oder religiösen Gehalten hebt der Oheim in erster Linie Gegenstände von historischem Wert auf. In der Galerie hängen ausschließlich Porträts historisch verbürgter Personen, von denen der Oheim sogar »Reliquien« (FA 10, 341) aufbewahrt. In der Hauptsache aber werden Texte, die als Sprüche über den Türen angebracht oder als Manuskripte zu Heften gebunden sind, überliefert. Das Schloss präsentiert sich dergestalt als Sammelstelle für »Heiltümer vergangener Zeiten« (FA 10, 341). Die sakralisierende Rede von Reliquien und Heiligtümern darf allerdings nicht darüber hinwegtäuschen, dass alle Objekte einer wissenschaftlichen Prüfung unterzogen worden sind. Das Schloss des Oheims bietet eine an strengen Maßstäben orientierte Sammlung von Bildern und Texten, die sich der Archivierung und gezielten Traditionsbildung verschrieben hat. Fast alles, was war, soll hier noch zu finden sein.

Über diesen Oheim wird Wilhelm die Bekanntschaft von Makarie machen, deren Textarchiv als organisierendes Zentrum des Romans gelten kann, verwahrt sie doch ebenjene Korrespondenzen, Tagebücher, Gesprächsprotokolle, Erzählungen und Spruchsammlungen, aus denen der Text der Wanderjahre - so will es die romaninterne Fiktion - gebaut worden ist. Gemäß dieser Konstruktion bestehen die Wanderjahre aus einer Vielzahl von Schriftzeugnissen unterschiedlicher Urheber. Diese Papiere werden von einem namenlos bleibenden Redaktor verwaltet, der sich beim Auswählen und Anordnen des Materials immer wieder kommentierend zu Wort meldet. Die Beschreibung dieser höchst eigenwilligen Erzählstruktur hat in der Forschung eine produktive Debatte eröffnet. Bezeichnet Erich Trunz in seinem Kommentar zu Wilhelm Meisters Wanderjahren die Romanform noch schlicht als »breiten gewichtigen Rahmenbericht « mit »einer Reihe von eingestreuten Novellen «, ${ }^{2}$ so haben Volker Neuhaus, Ehrhard Bahr, Safia Azzouni, Martin Bez und Stefan Willer mit den Stichworten Archivroman, Archivfiktion, kollektive Autorschaft, Archivpoetik und Archivpolitik in der Diskussion unterschiedliche Akzente gesetzt. ${ }^{3}$ Das »Archiv

\footnotetext{
1 Johann Wolfgang von Goethe, Wilhelm Meisters Wanderjahre oder Die Entsagenden, Zweite Fassung 1829, in: Ders., Sämtliche Werke. Briefe, Tagebücher und Gespräche, 40 Bände, hrsg. Hendrik Birus, Dieter Borchmeyer u. a., Abteilung I, Bd. 10, hrsg. Gerhard Neumann, Hans-Georg Dewitz, Frankfurt a. M. 1989, 261-774, hier: 341. Texte aus dieser Ausgabe werden im Folgenden zitiert unter der Sigle FA Band, Seite.

2 Erich Trunz, »Kommentarteil«, in: Johann Wolfgang von Goethe, Wilhelm Meisters Wanderjahre, in: Ders., Werke. Hamburger Ausgabe, hrsg. Erich Trunz, Bd. 8, München 1981, 527.

3 Volker Neuhaus, »Die Archivfiktion in >Wilhelm Meisters Wanderjahren««, Euphorion 62 (1968), 13-27; Gonthier-Louis Fink, »Tagebuch, Redaktor und Autor. Erzählinstanz und Struktur in Goethes >Wilhelm Meisters Wanderjahre«", Recherches Germaniques 16 (1968), 7-54; Ehrhard Bahr, The novel as archive.
} 
als Textverfahren $«{ }^{4}$ dies kann mittlerweile wohl als Konsens gelten, bietet nicht nur eine gleichsam dokumentarische Beglaubigung des Erzählten, sondern verantwortet auch eine Erzählform, die lose gefügt und fragmentarisch, nur bedingt kausallogisch und scheinbar kontingent organisiert ist.

Eine weitere Konsequenz dieser kompilatorischen Struktur ist indes noch nicht ausreichend bedacht worden. Denn in der Montage unterschiedlicher Textsorten wird nicht nur ein Spiel mit fiktiven Ebenen, medialen Bedingungen und variablen Autorschaften, sondern auch ein Spiel mit der Zeit getrieben. Betrachtet man das Verhältnis der sogenannten Rahmenhandlung des Romans zu den eingerückten Geschichten genauer, dann hat man es mit wenigen Ausnahmen eigentlich nicht mit Sprüngen zwischen verschiedenen fiktiven Welten, sondern mit Sprüngen in der Zeit zu tun. Wenn Figuren, die in den eingelegten Erzählungen eingeführt werden, später auch in der Haupthandlung um Wilhelm auftreten, dann lässt sich dies mit den von Gérard Genette geprägten narratologischen Begriffen nicht als Metalepse, also als »Übergang von einer narrativen Ebene zur anderen $\ll,{ }^{5}$ sondern präziser als Analepse beschreiben.

Tatsächlich handelt es sich bei der Mehrzahl der durch eigene Überschriften abgesetzten Erzählungen offensichtlich nicht um Rahmensprünge, sondern um Vorgeschichten. In der autobiografischen Erzählung von Joseph dem Zweiten, in Lenardos Erinnerung an Das nußbraune Mädchen, in der vom Barbier zum Besten gegebenen märchenhaften Geschichte Die schöne Melusine, in St. Christophs kurzem Schwank Die gefährliche Wette bis hin zur letzten Erzählung Nicht zu weit ist dies unmittelbar erkennbar: Hier erzählen gerade aufgetretene Figuren aus ihrer eigenen Vergangenheit, um sich in Gestalt dramatisch verdichteter, novellistisch anmutender Narrative vorzustellen. Wo sie dies nicht selbst in der Ich-Form tun, etwa in der Erzählung Nicht zu weit, nimmt ihnen der Redaktor die Aufgabe ab. Als Analepse entpuppt sich auch die drei Kapitel des zweiten Buchs umfassende Erzählung Der Mann von funfzig Jahren, deren Protagonisten nur zwei Kapitel später mit Wilhelm einige

The genesis, reception, and criticism of Goethe's Wilhelm Meisters Wanderjahre, Columbia, SC 1998; Safia Azzouni, Kunst als praktische Wissenschaft. Goethes >Wilhelm Meisters Wanderjahre und die Hefte `Zur Morphologie`, Köln, Weimar, Wien 2005; Martin Bez, Goethes >Wilhelm Meisters Wanderjahre ‘. Aggregat, Archiv, Archivroman, Berlin 2013; Stefan Willer, »Archivfiktionen und Archivtechniken in und an Goethes >Wanderjahren««, in: Daniela Gretz, Nicolas Pethes (Hrsg.), Archiv/Fiktionen. Verfahren des Archivierens in Literatur und Kultur des langen 19. Jahrhunderts, Freiburg i.Br. 2016, 109-127.

4 Bez (Anm. 3), 19.

5 Gérard Genette, Die Erzählung, München ${ }^{2}$ 1998, 167. Genettes Diskussion der Analepse beruht auf den beiden Unterscheidungskriterien der Reichweite und des Umfangs. Reicht ein narrativer Sprung vor den Beginn der Basiserzählung zurück, so spricht Genette von einer externen Analepse, spielt sie sich innerhalb der Zeit der Basiserzählung ab, so nennt er dies interne Analepse. Auf dieser Grundlage trifft er eine weitere, allerdings nicht unproblematische Unterscheidung. Als heterodiegetische interne Analepse bezeichnet Genette einen Strang der Geschichte, der sich von dem der Basiserzählung unterscheidet, etwa in Gestalt von Vorgeschichten neu eingeführter Personen. Inwiefern diese von homodiegetischen Analepsen, die den Handlungsstrang der Basiserzählung betreffen, zu unterscheiden sind, ist aber fraglich - gehören die neu eingeführten Personen doch, sobald ihre Vorstellung geleistet wurde, ebenfalls zur Basiserzählung. Produktiver hingegen ist die Unterscheidung der homodiegetischen internen Analepsen in repetitive Analepsen, in denen etwas noch einmal (anders) erzählt wird, sowie kompletiven Analepsen, die nachträglich eine bereits zuvor markierte Lücke schließen. Das Verfahren der Ellipse, also der Auslassung, des Überspringens und Verschweigens, geht in den Wanderjahren Hand in Hand mit der nachträglichen Vermittlung zunächst verschwiegener Ereignisse. 
Tage an einem oberitalienischen See verbringen und schließlich in den letzten Kapiteln an den Paarbildungen und Reisebewegungen zwischen Europa und Amerika teilnehmen. ${ }^{6}$

Das Verfahren eines achronologischen Erzählens, das in Bezug auf die novellistischen Einlagen als leicht durchschaubare nachträgliche Motivation erscheint, gewinnt mit Blick auf die um Wilhelm und Lenardo gruppierte Handlung an Komplexität, werden chronologisch-lineare Verläufe doch gerade im vermeintlichen Rahmen des Romans verschleiert und durchkreuzt. Dies gilt zuallererst für die wenigen Handlungsschritte, die sich noch zu einer Lebensgeschichte des titelgebenden Protagonisten fügen könnten, mithin für Wilhelms Entscheidung, Arzt zu werden, für die Beratungen über diese Entscheidung mit Mitgliedern der Turmgesellschaft sowie für seine tatsächlich unternommene Ausbildung. Im vierten Kapitel des ersten Buchs wird ein Gespräch erwähnt, in dem Wilhelm dem Freund Montan, in den Lehrjahren noch als Jarno bekannt, von seinem Berufswunsch berichtet. Wilhelm zeigt JarnoMontan ein geheimnisvolles »Besteck«, das der Redaktor weiter verrätselt:

»Was es aber gewesen, dürfen wir an dieser Stelle dem Leser noch nicht vertrauen, so viel aber müssen wir sagen, dass sich hieran ein Gespräch anknüpfte, dessen Resultate sich endlich dahin ergaben, dass Wilhelm bekannte: wie er schon längst geneigt sei, einem gewissen besondern Geschäft, einer ganz eigentlich nützlichen Kunst sich zu widmen.«(FA 10, 299)

Wilhelms Entscheidung ist also zu Beginn des Romans »längst« gefallen, und Montan soll lediglich bei der Aufhebung des Wandergebots behilflich sein, damit Wilhelm mit seiner Ausbildung beginnen kann. Im neunten Kapitel des zweiten Buchs treffen beide nach einer »Zwischenrede«, die sich an »eine Pause und zwar von einigen Jahren « (FA 10, 515) anschließt, wieder aufeinander. Wilhelms anatomische Versuche, die in dieser Pause stattgefunden haben, werden aber erst im dritten Buch in einer gerafften Ich-Erzählung nachgeliefert. Wilhelms Begründung seiner Ausbildungsentscheidung findet sich in der am weitesten zurückreichenden Vorgeschichte. Im letzten Kapitel des zweiten Buchs ist ein Brief Wilhelms an Natalie eingerückt, in dem Wilhelm der Geliebten »eine der frühesten Jugendgeschichten « (FA 10, 542) anvertraut. Die Geschichte vom Bad mit dem schönen Fischerknaben und von dessen Ertrinkungstod überschreibt die Kindergeschichte der Lehrjahre, also die an Mariane adressierte Erinnerung ans Puppentheater und an die daraus resultierenden Wünsche. Nicht nur werden innerhalb der Wanderjahre entscheidende biografische Schritte des erwachsenen Wilhelm Meister in signifikanten Zeitversetzungen erzählt. Mit der nachgetragenen Jugendgeschichte greifen die Wanderjahre sogar noch vor ihren eigenen Anfang in den zeitlichen Bereich des Vorgängerromans der Lehrjahre zurück und verschaffen dem Protagonisten einen konkurrierenden, erst

\footnotetext{
6 Als Eröffnung einer weiteren fiktiven Welt statt eines Wechsels der Zeitebenen können lediglich die ersten beiden novellistischen Einlagen gelten, also Die pilgernde Törin (1. Buch, 5. Kapitel) und Wer ist der Verräter (1. Buch, 8. und 9. Kapitel). In allen anderen Binnenerzählungen werden die Vorgeschichten von Figuren präsentiert, die auch in der Haupterzählung auftreten. Dies entspricht einer Erzählregie, in der sich die eingelegten Erzählungen erst im Fortschritt der Romanlektüre als nachgeholte Vorgeschichten entpuppen.
} 
im Rahmen der Wanderjahre aktivierten Handlungsmotor. ${ }^{7}$ Die narrative Darstellung der auf Wilhelm bezogenen Ereignisreihe ist also von steten Vor- und Rückgriffen geprägt.

Auch Lenardos Geschichte wird in vorgreifenden Andeutungen, wiederholten Aufschüben und nachgereichten Rückgriffen präsentiert. Unter dem Titel Das nu $\beta$ braune Mädchen im ersten Buch begonnen, erhält die Geschichte um Lenardo und Susanne erst im dritten Buch die partielle Auflösung. Dies geschieht in Form eines Tagebuchs, dessen Schreibsituation zwar dramatische Unmittelbarkeit suggeriert, dessen berichteter Inhalt zum Zeitpunkt der Mitteilung aber bereits in einer unbestimmten Vergangenheit liegt. Über die Einbettung dieser Erzählung wird später noch mehr zu sagen sein - vorerst lässt sich sowohl für die Geschichten Wilhelms als auch Lenardos festhalten, dass gerade jene Geschehnisse, von denen die Protagonisten unmittelbar betroffen sind, nicht chronologisch fortschreitend, sondern in zurückgreifenden Zeitschleifen entwickelt werden. Der Roman erzählt nicht nur eine, sondern mehrere Vorgeschichten. Das erste Buch bringt die Vorgeschichte vom jungen Lenardo und dem >nußbraunen Mädchen<, das zweite Buch die Vorgeschichte von Wilhelms Erlebnis mit dem Fischerknaben. Hier greift der Roman auf gleichsam vorgeschichtliche Ereignisse zurück, die sich vor dem Einsatz der Romanhandlung zugetragen haben. Das dritte Buch entwickelt deren Folgen wiederum in einer signifikanten Zeitversetzung. Insofern Wilhelms Ausbildung und Lenardos Wiedersehen mit Susanne in internen Analepsen präsentiert werden, reichen sie zwar nicht vor den Anfang des Romans zurück, wohl aber vor den in der Chronik des Erzählten jeweils erreichten Zeitpunkt. Hier wird das Geschehen in Gestalt von Vorgeschichten vergegenwärtigt.

Diese erste Strukturbeschreibung soll zeigen, wie zentral das Zeitmanagement des Unterbrechens und Überspringens, des Hinauszögerns und Nachtragens für die Erzählordnung der Wanderjahre ist. Die dem Roman zugrunde liegende Fiktion eines Archivs von zu sichtenden und zu selektierenden Papieren ermöglicht es dem als Redaktor firmierenden Erzähler, sich vom Leitseil der Chronologie zu lösen und zeitlich aufeinander folgende Episoden umzusortieren. Die auf Tradition, Überlieferung und Bewahrung ausgerichtete Funktion des Archivs übersetzt sich dabei in eine Erzählstruktur, die durch die Umstellung der Ereignisse strenggenommen Geschichten in Vorgeschichten, Vergangenes in Vorvergangenes verwandelt. Ausgehend von diesem Befund soll hier nach den Prämissen und Effekten dieses Erzählens gefragt werden. Welche Formmodelle rufen die Wanderjahre auf? Welche Konsequenzen hat das Erzählen in Vorgeschichten? Und in welchem Zusammenhang steht die Temporalstruktur des Textes zu Zeitreflexionen, sei es zu Modellierungen einer Zeit der Geschichte oder einer Zeit der Natur?

Die letzte Frage impliziert einen Leitgedanken der hier vorgestellten Lektüre. Bei den Wanderjahren handelt es sich um ein Erzählprojekt, das sich intensiv mit dem Verhältnis von Altem und Neuem befasst. Dies betrifft nicht nur die recht genau erforschte Auseinandersetzung mit Phänomenen gesellschaftlicher Modernisierung

\footnotetext{
7 Zur Gestaltung von Vorgeschichten in Wilhelm Meisters Lehrjahren, die sich auch als Arbeit am heliodorischen Modell beschreiben lässt, vgl. Cornelia Zumbusch, »Nachgetragene Ursprünge. Vorgeschichten im Bildungsroman (Wieland, Goethe und Stifter)«, Poetica 43/3-4 (2011), 267-299.
} 
wie etwa den Frühformen der Technisierung und Industrialisierung, ${ }^{8}$ sondern auch die im Roman verhandelte Frage nach den jeweiligen Möglichkeiten der Bewahrung und Restitution von Vergangenem oder aber des radikalen Neuanfangs unter den Bedingungen beschleunigter Veränderungsraten. Das analeptische Erzählen, so lautet die im Folgenden zu belegende These, bietet eine hervorragende Möglichkeit, um diese Problematik nicht nur in der diskursiven Auseinandersetzung mit Modernisierungsphänomenen, sondern auch in der Erzählform selbst abzubilden. Denn wie über vieles andere herrscht auch über das Verhältnis von Altem und Neuem im Roman Dissens.

Ist das Alte eine Entwicklungsstufe des Neuen, die das Gegenwärtige besser verstehen hilft? Diese Position deutet sich an, wenn sich Wilhelm für seinen Sohn Felix eine gewisse Weitläufigkeit der Erziehung wünscht: »so schaut er doch zuletzt in seiner Zeit umher, und wie kann er die begreifen, wenn er nicht einigermaßen weiß, was vorhergegangen ist « (FA 10, 297). Oder wiederholt sich doch alles dergestalt, dass das Neue dem Alten kaum etwas hinzufügt - etwa wenn der Skeptiker und Widerspruchsgeist Jarno-Montan zu Wilhelm sagt: »Dort hinabwärts liegt eine neue Welt vor dir, aber ich will wetten, es geht darin zu, wie in der alten hinter uns « (FA 10, 291). Das in den Wanderjahren erprobte analeptische Erzählen dient, so soll hier argumentiert werden, der Sichtbarmachung und Bearbeitung dieses Problems. Dem Versuch, dies in der Kommentierung einiger Episoden und den dort wirksamen Strukturmotiven aufzuzeigen, ist der Hauptteil der folgenden Ausführungen gewidmet (III). Der letzte Teil bringt die Temporalstruktur des Romans mit einigen Zeitreflexionen aus Goethes geologischen Studien und das Erzählen in Vorgeschichten mit dem Prinzip einer Urgeschichte der Erde in Verbindung (IV). Vorangestellt sind aber zunächst Überlegungen zur Vorgeschichte als Erzählprinzip (II).

\section{II.}

Was eine Vorgeschichte ist, bestimmt sich nicht so sehr aus dem erzählten Gegenstand, als vielmehr aus dem Modus seiner narrativen Darstellung. Ein Ereigniszusammenhang wird dann zur Vorgeschichte, wenn er aus dem chronologischen Ablauf einer Geschichte, narratologisch gesprochen aus der histoire, herausgelöst und an späterer Stelle der Erzählung, dem discours, nachgetragen wird. Wer eine Vorgeschichte erzählt, geht vor einen bereits gesetzten Anfang zurück und eröffnet den Blick auf eine Zeit, die nun als Vorzeit erscheint. Im Modus der Vorgeschichte wird somit Vergangenheit in eine doppelt entrückte Vorvergangenheit verwandelt. Rückgriffe vor den Beginn der Erzählung machen zugleich offenkundig, dass jede

\footnotetext{
8 Vgl. hierzu die Untersuchungen von Franziska Schößler, Richard Meier, André Lottmann und Günter Sasse, die mit unterschiedlichen Schwerpunkten die im Roman geleistete Beobachtung von Modernisierungsphänomenen herausgearbeitet haben. Franziska Schößler, Goethes Lehr- und Wanderjahre. Eine Kulturgeschichte der Moderne, Tübingen, Basel 2002; Richard Meier, Gesellschaftliche Modernisierung in Goethes Alterswerken >Wilhelm Meisters Wanderjahre und >Faust II<, Freiburg i.Br. 2002; André Lottmann, Arbeitsverhältnisse. Der arbeitende Mensch in Goethes Wilhelm Meister-Romanen und in der Geschichte der Politischen Ökonomie, Würzburg 2011; Günter Sasse, Auswandern in die Moderne. Tradition und Innovation in Goethes Roman >Wilhelm Meisters Wanderjahre`, Berlin, New York 2010.
} 
Anfangssetzung etwas Vorangegangenes abgeschnitten, abgeschattet und dem Blick entzogen hatte. Wenn also Ereignisse als Vorgeschichten präsentiert werden, dann wird das, was zunächst wie ein Anfangspunkt aussah, als eine Grenzlinie kenntlich, die das Kontinuum des Geschehenen durchschneidet und in ein Davor und ein Danach trennt. Das Erzählen von Vorgeschichten eignet sich deshalb besonders gut dazu, Erfahrungen des Sprunghaften und Diskontinuierlichen zu artikulieren und auch selbst zu provozieren.

Die Aufteilung des Geschehens auf eine zunächst begonnene Hauptgeschichte und später eingeholte Vorgeschichten gehört ins Basisrepertoire des epischen Erzählens. Wie bereits Horaz vermerkt, lässt sich nicht nur ab ovo, sondern auch medias in res einsteigen, wobei Letzteres die ungleich glanzvollere Variante darstelle. ${ }^{9}$ In der spätmittelalterlichen Rhetorik wird dieser Umstand unter der Bezeichnung der ordo artificialis aufgenommen, die anders als die ordo naturalis in das chronologische Zeitgefüge eingreift. Auch hier sei die künstliche Ordnung diejenige, um die sich die Dichtung, wenn sie kunstvoll sein will, zu bemühen habe. Späteres früher zu bringen, das Letzte zum Ersten zu machen - darin läge die wahre Kunst der Komposition. ${ }^{10}$ Goethe und Schiller nutzen diese Beobachtung, um die Eigenheiten der dramatischen und der epischen Darstellung voneinander abzusetzen. Anders als die stets voraneilende dramatische Darstellung könne der epische Erzähler pausieren, verzögern und vor allem: er könne in der Zeit zurückgreifen. Diese Möglichkeit der retrograden Darstellung gehört zum proprium des Epischen. Denn während das bloße Retardieren, also das Aufhalten der Handlung, auch im Drama stattfinden könne, sei die $»\left[\right.$ r] ückwärtsschreitende ${ }^{11}$ Darstellung allein dem Epos vorbehalten. ${ }^{12}$

Der medias in res-Einstieg, die ordo artificialis wie auch das retrograde Erzählen haben von Horaz bis Goethe ihr bevorzugtes Formmodell in Homers Odyssee. Tatsächlich ist gerade von dem, was man als die Abenteuer des Odysseus kennt, also die Begegnungen mit den Lotophagen, mit dem Kyklopen Polyphem, mit Kirke und Kalypso sowie Odysseus' Gang in die Unterwelt, erst im neunten bis zwölften Gesang die Rede. Die ersten Gesänge hingegen etablieren eine um Odysseus’

\footnotetext{
9 Quintus Horatius Flaccus, Ars Poetica/Die Dichtkunst, Lateinisch/Deutsch, übers. und hrsg. Eckart Schäfer, Stuttgart 1972, 13.

10 Geoffrey of Vinsauf, Poetria nova [1199/1215], übers. Margaret F. Nims, Toronto 1967, 19.

11 Johann Wolfgang von Goethe und Friedrich Schiller, »Über epische und dramatische Dichtung «, FA 18, 445-447, hier: 444.

12 Wie an dieser Stelle leider nicht ausführlich entwickelt werden kann, schließt die Erzählforschung von Robert Petsch, Günther Müller und Eberhard Lämmert bis Gérard Genette in zentralen Punkten an diese frühen Reflexionen aus Poetik und Rhetorik an. Einig sind sich die meisten Ansätze darin, dass Verstöße gegen die Handlungschronologie als spezifisch epische oder erzählerische Mittel und damit als Differenzkriterium zur dramatischen Darstellung gelten können. Die willkürliche Umstellung der natürlichen Zeitabläufe gilt dabei als Garant einer Artifizialität, mit der sich die kunstvolle Erzählung vom bloß chronikalischen Bericht abhebt. In der Narratologie des 20. Jahrhunderts, insbesondere bei Lämmert und Genette, erweisen sich die Rückgriffe in der Zeit darüber hinaus als Zonen intensivierter Bedeutungserzeugung oder einer reflexiven Distanzierung vom bereits Erzählten, mithin als Anlass der immanenten Selbstbezüglichkeit. In Genettes Lektüre der recherche du temps perdu etwa wird die Analepse zum herausgehobenen Moment der Reflexion des Erzähltextes auf sich selbst. Robert Petsch, Wesen und Formen der Erzählkunst (1934), 2. Aufl., Halle 1942, 162-180; Günther Müller, Morphologische Poetik, Tübingen 1968, 299-396; Eberhard Lämmert, Bauformen des Erzählens (1955), Stuttgart 1993, 99-137; Genette (Anm. 5), 39-41.
} 
Sohn Telemach gruppierte Handlung, in der die Situation der den Hof und Penelope belagernden Freier im kritischen Moment des zwanzigsten Jahres von Odysseus' Abwesenheit entwickelt wird. Dieses Problem wird dann in den letzten zehn Gesängen mit Odysseus' Eintreffen in Ithaka und dem ebenfalls ausführlich erzählten Mord an den Freiern $>$ gelöst $<.{ }^{13}$ Die der Heimkehr vorangehenden zehn Jahre der Irrfahrt finden sich somit nicht am Anfang, sondern in der Mitte des Epos, wo sie in einer analeptischen Schleife zusammengezogen präsentiert werden. Das, was man für den eigentlichen Gegenstand der Odyssee halten könnte, ist dadurch nur als Vorgeschichte greifbar. ${ }^{14}$ Hervorzuheben ist an diesem Modell, dass die Rückschritte in der erzählten Zeit in den Raum des Märchenhaften und Mythischen als den eigentlich produktiven Zonen des Erzählens führen. ${ }^{15}$

Nun finden sich in den Wanderjahren motivische Anspielungen auf die Odyssee, die vielleicht nicht nur als beliebige Fäden im dichten Gewebe des mythologischen Subtexts zu lesen sind, sondern die auch Aufschluss über die narrative Matrix der Wanderjahre geben. Zunächst ist offensichtlich, dass die Geschichte um Lenardo und Wilhelm von einer aufgeschobenen Rückkehr erzählt. Ausgelegt wird diese Spur in den ersten Kapiteln, als sich Wilhelm mit dem Familienkreis des Oheims bekannt macht und dort erfährt, dass der Sohn Lenardo aus unerklärlichen Gründen seine längst fällige Heimkehr aufschiebt. Es folgen Briefe der Nichten Hersilie und Juliette sowie ihrer Tante Makarie, die über die Motive des Abwesenden beraten. Ein ebenfalls eingerückter Brief Lenardos an die Tante erklärt recht knapp, dass er erst Auskunft über ein Mädchen haben möchte, deren Familie zur Finanzierung seiner eigenen Reise die Pacht gekündigt worden war. Hier ist offensichtlich eine Schuld abzutragen oder zumindest Kenntnis von der daraus erwachsenen Situation zu gewinnen. Als Lenardo das Mädchen im dritten Buch als verwitwete Leiterin eines Webereibetriebs wiederfindet, kommt sie ihm vor wie »Penelope unter der Mägden«(FA 10, 700). Der Vergleich mit der treuen Ehefrau des Odysseus, die dem Werben der Freier durch die nachts stets aufgelöste Webarbeit klug auszuweichen weiß, verweist auf Susannes Situation zwischen der Trauer um ihren verstorbenen Ehemann und der Aussicht auf eine pragmatische Verlobung mit dem Gehülfen eine mögliche Verbindung mit Lenardo wird allenfalls angedeutet.

\footnotetext{
$13 \mathrm{Zu}$ Odysseus als Kriegsheimkehrer und insbesondere zum Zusammenhang von Rückkehr und Gewalt vgl. Eva Esslinger, »Heimkehr eines Kriegsveteranen. Einleitende Überlegungen zu Homers Odyssee«, DVjs 92/2 (2018), 92-127.

14 Mit Ausnahme der prinzipiell einschlägigen Formgesetze des homerischen Epos von Harald Patzer, der die Odyssee für streng linear erzählt hält, ist man sich in der Forschung einig über die achronologische Erzählform. Harald Patzer, Die Formgesetze des homerischen Epos, Stuttgart 1996, 88-97. Zum Konsens über den medias in res-Einstieg und die zurückgreifende Konstruktion sei hier auf drei Monografien mit einführendem Charakter verwiesen: Uvo Hölscher, Die Odyssee. Epos zwischen Märchen und Roman, München 1988, 45, Joachim Latacz, Homer. Der erste Dichter des Abendlands, München, Zürich ${ }^{2} 1989$, 174 und Gustav Adolf Seeck, Homer. Eine Einführung, Stuttgart 2004, 106.

15 In etwas anderer Akzentuierung steht das Verhältnis von Epos und Märchen, Geschichte und Mythos im Zentrum der Darstellung von Uvo Hölscher, insofern er für die aus märchen- und legendenhaften Geschichten gebaute Erzählung der Irrfahrten geltend macht: »Was als einfache Geschichte in einer zeitlosen Sphäre spielt, wird in der Dichtung Erinnerung der Vorzeit: Geschichte im prägnanten Sinn«. Hölscher (Anm. 14), 159. Wie sich an der Odyssee zeigen lässt, betrifft dies nicht (nur) das Verhältnis von Geschichte und Mythos, sondern bezeichnet auch den Ursprung des fiktionalen Erzählens.
} 
Aufschlussreich ist der Vergleich mit der Odyssee insbesondere im Blick auf die Organisation dieser Erzählstränge. Denn was bei Homer als Verflechtung einer eigentlichen Odyssee mit der Telemachie diskutiert worden ist, findet sich in den Wanderjahren in der Parallelführung der Handlungen um Wilhelm und Lenardo. So erhält Wilhelm wie Telemach den Auftrag, die Wiederkehr des Lenardo vorzubereiten und die dafür nötigen Erkundigungen einzuziehen. Die in den Wanderjahren vorgelegte Variation auf dieses Modell ist allerdings komplexer. Zum einen ist die Vater-Sohn-Konstellation der Odyssee auf Wilhelm und Felix verlegt, die, ganz anders als Odysseus und Telemach, gemeinsam in den Roman eintreten, um sich dann erst zu trennen und einander zuletzt wieder zu finden. Zum anderen ist Wilhelms Reisebewegung ihrerseits aufgefaltet in die auf Lenardo bezogene diplomatische Mission und in die Geschichte seiner eigenen Ausbildung.

Was die Wanderjahre und die Odyssee nicht zuletzt verbindet, ist ihr Einstieg medias in res. Die Odyssee setzt mit dem von den Göttern gefassten Beschluss ein, den auf der Insel der Kalypso gestrandeten Odysseus nach 19 Jahren der Abwesenheit endlich nach Hause zu lassen. Die Odyssee gewinnt ihren Anfang also aus einer entscheidenden Wendung der Ereignisse. In den Wanderjahren ist der Wendepunkt in die Raumsymbolik verlagert. Die Wanderjahre beginnen »an grauser, bedeutender Stelle, wo sich der steile Gebirgsweg um eine Ecke herum schnell nach der Tiefe wendete (FA 10, 263). Mit den beiden implizierten Wendungen, die um eine Wegbiegung und zugleich in die Tiefe führen, ist das Motiv des Richtungswechsels noch herausgehoben und der Eingang in den Roman als Schwelle zu einem noch nicht überschauten Bereich gestaltet. Wie die Odyssee beginnen auch die Wanderjahre nicht an einem Anfangs-, sondern an einem Wendepunkt. Weit intensiver als die Odyssee reflektieren die Wanderjahre ihr Verfahren des Rückwärtserzählens in Strukturmotiven, die Vergangenheit und Möglichkeiten der Wiederkehr evozieren.

Schon Wilhelms erster Blick auf »Sankt Joseph« und seine Familie, deren Bekanntschaft er im ersten Kapitel macht, ist ein Blick in die Tiefe: »Neugierig stand er auf und blickte von seiner Stelle nach der Tiefe hin« (FA 10, 265). Beim vorläufigen Abschied hört Wilhelm den Gesang der Familie »aus der Tiefe« (FA 10, 267). Indem er ihnen folgt, führt sein Weg in den Roman nicht in eine verheißungsvolle Zukunft, sondern in eine eigenartig unbestimmbare Zeitzone. Wilhelm spricht die Josephsfamilie bei der ersten Begegnung als »Geister « an und assoziiert sie mit den Motiven des Todes wie auch der Wiederkehr. ${ }^{16}$ Für Wilhelms eigene Reise in diese »düstere Tiefe« (FA 10, 269) bietet der Text eine radikale Deutung an: »der Wirt«, so notiert Wilhelm in der Herberge beim Packen seiner Sachen, »räumt schon wieder auf in meiner Gegenwart, eben als wenn ich hinweg wäre, wie gefühllose unvorsichtige Erben vor dem Abscheidenden die Anstalten, sich in Besitz zu setzen, nicht verbergen« (FA 10, 269). Indem der Roman den Beginn als symbolischen Tod seines Protagonisten inszeniert, deutet er an, dass sich alles Folgende in einem schwer datierbaren Schwellenbereich aus Gewesenem und seiner geisterhaften Wiederkehr abspielen wird.

16 Zur Motivik der geisterhaften Wiederkehr vgl. Barbara Naumann, »Geistererscheinungen. Wiederholung und Symbolisierung in Goethes Roman >Wilhelm Meisters Wanderjahre «, in: Carola Hilmes, Dietrich Mathy (Hrsg.), Dasselbe Noch einmal. Die Ästhetik der Wiederholung, Wiesbaden 1998, 38-62. 
Das Gespräch mit Joseph eröffnet Wilhelm am nächsten Tag denn auch mit der Frage, ob und auf welche Weise das Vergangene wiederkehren kann: »macht mich mit eurer Geschichte bekannt, damit ich erfahre, wie es möglich war, daß ohne Spielerei und Anmaßung die Vergangenheit sich wieder in euch darstellt, und das was vorüberging, abermals herantritt« (FA 10, 272). Das Motiv des »abermals« als einer Wiederholung des Vergangenen wird am Beispiel des zweiten Joseph in zwei Organisationsmodellen durchgespielt. Erstens im Modell der restitutio, der baulichen Wiederherstellung von Gewesenem, und zweitens im Modell der imitatio, der Nachahmung oder Nachfolge, in der die Bewohner eines Sakralbaus Vergangenes wieder zum Leben erwecken. Joseph hat eine verfallene Kapelle mit einem Bilderzyklus vom Leben des heiligen Joseph restauriert und für sich und seine Familie zum täglichen Leben eingerichtet. Im daraus resultierenden bunten Nebeneinander des Religiösen und Säkularen, des Alten und Neuen bietet diese Kapelle ein in den Raum projiziertes Zeitmodell. Das Nachleben des christlichen Mythos im häuslichen Alltag führt die Gleichzeitigkeit von historisch eigentlich Ungleichzeitigem vor Augen.

Dieses Spiel mit den Zeiten verwischt die Unterscheidung zwischen dem, was zuerst kam, und dem, was nachfolgte. Wenn Wilhelm die Malereien an den Kapellenwänden als »Wiederholung des gestrigen lebendigen Bildes hier an der Wand« (FA 10, 272) bezeichnet, dann ist das Leben des zweiten Joseph zum Original, das sakrale Urbild hingegen zu dessen Wiederholung geworden. Hier sind geläufige chronologische Vorstellungen umgekehrt. Die Kapelle ist für Joseph selbst der Ort der Nachfolge als einem Sich-Angleichen und Ähnlich-Werden: »Es lag eine unwiderstehliche Neigung in mir diesem Heiligen nachzufolgen; und da sich ähnliche Begebenheiten nicht leicht herbeirufen ließen, so wollte ich wenigsten von unten auf anfangen, ihm zu gleichen « (FA 10, 279). Die Episode um Sankt Joseph veranschaulicht ein Programm, das als paradigmatisch für die Chronopoetik des Romans gelten kann. Denn die Topologie des >von unten auf< als einem Fortschreiten vom Einfachen zum Komplexen bündelt Vorstellungen von räumlicher Tiefe, Bedeutungstiefe und Zeitentiefe in einer Weise, die in den Erzählreflexionen des Romans wiederaufgenommen wird.

Im Kapitel um Makarie meldet sich der Redaktor-Erzähler mit einer aphoristisch anmutenden Erklärung des Aphorismus zu Wort: »Resultate waren es, die wenn wir nicht ihre Veranlassung wissen, als paradox erscheinen, uns aber nötigen vermittels eines umgekehrten Findens und Erfindens rückwärtszugehen und uns die Filiation solcher Gedanken von weit her, von unten herauf wo möglich zu vergegenwärtigen « (FA 10, 389, Hvh. CZ). Das >von unten herauf< wird hier als der Ort der Veranlassungen, Gründe und Ursprünge lesbar, die sich nur im Rückwärtsgang erschließen lassen. Die Verknappungsform des Aphorismus soll zu einer Lektürepraxis anleiten, die insofern >rückwärtszugehen< hat, als sie den Weg des Denkens von seinen Ergebnissen zu deren Ursachen zurückverfolgt. Was hier für die in den Roman integrierte Spruchdichtung gelten soll, lässt sich auch als Bauplan des ganzen Romans lesen, folgt doch auch das Erzählschema der Wanderjahre nicht der kausallogischen Abfolge von Ursachen und Folgen, sondern gruppiert diese Abfolge um. Die anachrone Anordnung der Ereignisse führt dazu, dass Ergebnisse von 
Handlungsschritten immer wieder vor den zugrunde liegenden Ursachen, also den Handlungsgründen, präsentiert werden.

Aufschlussreich ist weiterhin die im Aphorismus auffällige Kopplung von >Finden und Erfinden<, die offenkundig macht, dass der retrograden Aufdeckung von Ursachen auch ein Moment des Fiktiven anhaftet. Auf die im Roman erzählten Vorgeschichten übertragen hieße dies, dass die in den Analepsen aufgewiesenen Handlungsgründe nicht nur als Gegenstand der Rekonstruktion, sondern auch als Ergebnis einer Konstruktion gelten müssen. Die nachgetragenen Vorgeschichten gehören womöglich nicht nur in den Bereich der Erinnerung, sondern ebenso in denjenigen der Erfindung. Vor diesem Hintergrund erschließt sich, warum sich die mit eigenen Überschriften abgesetzten Geschichten erst auf den zweiten Blick als analeptische Rückschritte in der Zeit zu erkennen geben. Indem Goethe sie als Erzählungen eigenen Rechts vom umliegenden Text abhebt, lässt er das, was Filiationen von weither sichtbar machen soll, als Fiktion in der Fiktion erscheinen. ${ }^{17}$ Die Zweideutigkeit von Finden und Erfinden, von analeptischer Vorgeschichte und novellistischer Binnengeschichte, verdeutlicht den prekären epistemischen Status zurückliegender und nur im Rückwärtsschreiten zu erreichender Ursachen: Das derart Gefundene könnte eben auch Erfundenes sein, nicht Faktum, sondern Fiktion. Welchen Zwecken folgt nun dieses im Roman nicht nur praktizierte, sondern auch reflektierte Erzählen in Vorgeschichten und welche Effekte zeitigt es?

\section{III.}

Die in den Wanderjahren entwickelte Spielart des analeptischen Erzählens hat entscheidende Konsequenzen für die Lesbarkeit des Textes. Das Verwischen der Grenzen zwischen einem Rückgriff in der Zeit und einem Wechsel auf eine andere fiktionale Ebene führt zu einer zeitlichen Desorientierung, der man sich beim Lesen der Wanderjahre kaum entziehen kann. Die Schwierigkeit, die erzählten Ereignisse auch nur grob zu datieren, ist im Roman nicht einfach nur in Kauf genommen, sondern wird ganz offensichtlich forciert. Deutlich wird dies an der zwischen der ersten und der zweiten Fassung der Wanderjahre bemerkbaren Tendenz, Hinweise auf konkrete zeitliche Abläufe zu tilgen. In der ersten Fassung trifft man den Redaktor immer wieder dabei an, wie er sich über die Chronologik der ihm vorliegenden Papiere den Kopf zerbricht. Dabei wird ihm »bemerklich, daß wir im Roman, eben wie es in der Weltgeschichte geht, an ungewisser Zeitrechnung leiden, und nicht ganz entschieden zu bestimmen vermögen was sich früher oder später ereignet « (FA 10, 162). Obwohl sich diese grundsätzliche Unbestimmtheit nicht auflösen lässt, bemüht sich der Redaktor um die exakte Rekonstruktion der zeitlichen Abläufe und Verhältnisse. Zwar sei »bei so vielen andern Blättern das Datum vernachlässigt«, ihm liege aber eine Landkarte vor, auf der »in gewisser Folge, mehrere Monatstage angeschrieben« (FA 10, 162) seien. Mithilfe dieser Karte hofft er die Intervalle von Wilhelms

17 Betrachtet man die Textgenese des Romans, so präsentiert sich dieser Zusammenhang umgekehrt, werden die seit 1808 geschriebenen und vorab publizierten Erzählungen doch erst durch die Integration in die Wanderjahre zu Vorgeschichten. 
Wanderbewegung nachvollziehen zu können. Diese Überlegungen des Redaktors, die den vertrauten Standard eines zeit- und chronologiebewussten Romanplots noch hochhalten, obwohl sie ihm nicht mehr folgen, sind in der zweiten Fassung von 1829 nicht mehr aufzufinden.

Ähnliches lässt sich auch bei der zeitlichen Einordnung der novellistischen Einlagen feststellen. Die Erzählung Der Mann von funfzig Jahren etwa wird in der ersten Fassung von Hersilie eingeleitet, indem sie dem Leser einen chronologischen Leitfaden an die Hand gibt. Sie schreibt an Wilhelm: »Ein halbes Jahr ist nun seit Ihrer Abreise verflossen, vor dem Ende des ganzen dürfen wir nicht hoffen Sie bei uns zu sehen« (FA 10, 104). Im nächsten Absatz macht sie Wilhelm auf zwei »allerliebste weibliche Wesen « aufmerksam, die sich soeben auf Reisen befänden. Aufschluss über die beiden Frauen soll Wilhelm durch die nachfolgend eingerückte Geschichte erhalten, in der sie als Nichte des Manns von funfzig Jahren und als schöne Witwe auftreten. Der als Vorgeschichte eingeführten Erzählung ist gleichwohl ein caveat mitgegeben: Erzählt wird in Der Mann von funfzig Jahren nicht die Geschichte der beiden Frauen, sondern »eine Geschichte, die sich einigermaßen auf sie bezieht; was daran wahr oder erdichtet ist, suchen Sie von ihnen selbst zu erfahren « (FA 10, 105). Einen ähnlichen Kunstgriff wie hier in der ersten Fassung der Wanderjahre hat Goethe bei der Einbettung der Novelle Die wunderlichen Nachbarskinder in den Roman die Wahlverwandtschaften - die ja ihrerseits als novellistische Einlage der Wanderjahre geplant waren - gewählt. ${ }^{18}$

Hersilies Orientierungshilfe, die gleichermaßen auf den analeptischen Charakter der Novelle wie auch auf den fiktiven Charakter der Vorgeschichte hinweist, ist in der zweiten Fassung der Wanderjahre umgeformt. Hier fungiert der Redaktor als rahmende Instanz, der die Erzählung mit dem Wunsch versieht: »Möge [...] am Ende deutlich werden, wie die Personen dieser abgesondert scheinenden Begebenheit mit denjenigen die wir schon kennen und lieben auf's innigste zusammengeflochten werden.« (FA 10, 433) Weder gibt es in der zweiten Fassung konkrete Zeitangaben, noch wird erwähnt, dass Wilhelm die Protagonistinnen der Erzählung treffen kann. Der Hinweis auf eine mögliche Verflechtung mit der Hauptgeschichte bleibt entsprechend vage. Was beide Fassungen verbindet, ist das offene Ende der Erzählung selbst, das nur die Zuspitzung des Konflikts, nicht aber dessen Lösung erzählt. Dieses aufgeschobene Ende wird erst stattfinden, nachdem Wilhelm den beiden Frauen begegnet ist. Im letzten Buch des Romans tauchen die Protagonisten in den generationsgerechten Paarungen auf: Der Mann von fünfzig Jahren ist mit der schönen Witwe liiert, seine Nichte Hilarie hat seinen Sohn Flavio geheiratet. Dieses auf-

\footnotetext{
18 Hier heißt es über Charlotte, die Geschichte sei ihr »bekannt«: »Diese Begebenheit hatte sich mit dem Hauptmann und seiner Nachbarin wirklich zugetragen, zwar nicht ganz so, wie sie der Engländer erzählte, doch war sie in den Hauptzügen nicht entstellt, nur im Einzelnen mehr ausgebildet und ausgeschmückt, wie es dergleichen Geschichten zu gehen pflegt, wenn sie erst durch den Mund der Menge und sodann durch die Phantasie eines geist- und geschmackreichen Erzählers durchgehen. Es bleibt zumeist alles und nichts wie es war.« Goethe, Die Wahlverwandtschaften, FA 8, 479. Die auffällige Parallele in Bezug auf den gleitenden Übergang vom tatsächlich vormals Geschehenen (»wirklich zugetragen«) über die erzählerisch kunstvolle Formgebung (»ausgebildet und ausgeschmückt«) bis zur vollkommenen Umkehrung (»nichts wie es war«) wäre an anderer Stelle genauer auszuarbeiten. Dabei wäre auch die im Folgenden noch zu besprechende traumatische Struktur der analeptischen Novellistik zu bedenken.
} 
geschobene Ende führt auf ein weiteres Charakteristikum der Vorgeschichten: Sie enthalten allesamt Ungelöstes. Oder wie es in der ersten Fassung heißt: Es sind »Erzählungen«, die »zugleich Auflösung und Abschluß fordern« (FA 10, 128). Dies betrifft ganz besonders die Vorgeschichten Lenardos und Wilhelms. ${ }^{19}$

Lenardo erzählt vom nußbraunen Mädchen, um sich von seinem schlechten Gewissen zu lösen: »wenn ich das Mädchen glücklich weiß, bin ich sie los« (FA 10, 409), so verspricht er. Dabei kommt das zurückliegende Geschehen zunächst nur lückenhaft zur Sprache. Warum dies so ist, führt Lenardo im Bild von einer Wunde vor Augen: »Ein lebhafter Eindruck ist wie eine andere Wunde; man fühlt sie nicht, indem man sie empfängt. Erst später fängt sie an zu schmerzen und zu eitern. [...] Es war ein unauslöschlicher Eindruck, der wohl von andern Bildern und Teilnahmen beschattet, verdeckt, aber niemals vertilgt werden konnte « (FA 10, 397). Ein erschütterndes Ereignis ist laut Lenardo eine andersartige, wohl keine physische, sondern eine psychische Wunde, deren Ursache weder korrekt erinnert noch komplett vergessen werden kann. Symptomatisch für diesen Verdeckungsprozess ist es, dass Lenardo zunächst einen falschen Namen erinnert und ein Mädchen namens Valerine aufsucht. Erst als Valerine den richtigen Namen des nußbraunen Mädchens ins Spiel bringt, kann sich Lenardo erinnern: »Kaum hatte Lenardo den Namen Nachodine gehört, so entsann er sich dessen vollkommen; aber auch mit dem Namen kehrte das Bild jener Bittenden zurück, mit einer solchen Gewalt, daß ihm das Weitere ganz unerträglich fiel.« (FA 10, 404) Bedenkenswert ist zuletzt eine Einschränkung, mit der Lenardo dem fehlerhaft Erinnerten den generischen Status der Geschichte abspricht. Er will Wilhelm »erzählen, was eigentlich keine Geschichte ist« (FA 10, 393). Der schmerzhafte Charakter der Erinnerung als Wunde (griech. trauma) scheint zu verhindern, dass dem Geschehenen eine konventionelle narrative Form gegeben werden kann. An der hier vorgeführten Abfolge von einem gewaltsamen schmerzhaften Eindruck, dessen Verdeckung und Verfälschung und schließlich seiner bruchstückhaften Wiederkehr hätte Freud seine Definition des Traumas abschreiben können. ${ }^{20}$ Ebenso schmerzhaft wie unabgegolten, so sei zumindest angedeutet, sind auch die in Wil-

\footnotetext{
19 Aber auch noch die märchenhafte Erzählung des Barbiers Rotmantel, der eingangs bekennt: »[...] daß ich eine zu erzählen habe, welche die bisherige weit übertrifft, und die, wiewohl sie mir schon vor einigen Jahren begegnet ist, mich noch immer in der Erinnerung unruhig macht, ja sogar eine endliche Entwicklung hoffen läßt.« (FA 10, 633). Noch der scheinbar ganz deplatzierte Schwank endet mit tiefer Kränkung, Wunde und Tod (FA 10, 664). Nicht zu weit endet mit der lakonischen Feststellung »der Schade war geschehen« (FA 10, 685).

20 Dies gilt weniger für Freuds frühe Traumatheorie im Rahmen der Ätiologie der Hysterie, als vielmehr für die spätere Konzeptualisierung des Traumas in Jenseits des Lustprinzips (1920) sowie Hemmung, Symptom und Angst (1926) - Texte, in denen vor allem die Gesetze der Wiederkehr in den Blick rücken. In diesem Zusammenhang wäre das, was Freud bereits in den Vorlesungen zur Einführung in die Psychoanalyse (1915-1917) ein »prähistorisches Ereignis« nennt und von einem tatsächlichen infantilen Ereignis unterscheidet, genauer auf die Zusammenhänge von individueller und kollektiver Vorgeschichte zu beziehen. Sigmund Freud, Vorlesungen zur Einführung in die Psychoanalyse, in: Ders., Gesammelte Werke. Chronologisch geordnet, hrsg. Anna Freud u. a. (London 1940), Frankfurt a. M. 1999, Bd. 11, hier: 376. Zum Status der Begriffe Vor- und Urgeschichte in Freuds kulturtheoretischen Schriften, insbesondere in Totem und Tabu, vgl. Cornelia Zumbusch, »Urgeschichte. Erzählungen vom Vorvergangenen bei Herder, Engels, Freud und Benjamin«, in: Tobias Döring, Michael Ott (Hrsg.), Urworte. Zur Archäologie erstbegründender Begriffe, München 2012, 137-153, hier: 145-148.
} 
helms Vorgeschichte produzierten Ereignisse. ${ }^{21}$ Einschneidend ist hier nicht nur die schnelle Abfolge der erotischen Erfahrung und der jäh darauffolgenden Erfahrung des gewaltsamen Todes des schönen und begehrten Knaben. In die Gegenwart reicht insbesondere der aus dieser Erfahrung abgeleitete Anspruch, sich selbst zum Retter zu machen.

Sowohl die Geschichte vom nußbraunen Mädchen als auch Wilhelms Erinnerung an den Tod des Fischerknaben gehen in ihrer Funktion weit über das hinaus, was Vorgeschichten aus narratologischer Sicht zugetraut wird. Sie stellen nicht nur Personen vor oder tragen Informationen nach, die man wissen muss, um die weitere Entwicklung des Plots zu verstehen. Wenn die Vorgeschichten der Wanderjahre ein Wissen vermitteln, dann eines, das im Zeichen einer Arbeit am Unerledigten steht. Sie lassen etwas hervortreten, was lange verborgen gehalten wurde, und in diesem Verborgenen umso stärker wirken konnte. Dazu passt auch, dass Wilhelm gleichsam in verdeckter Mission unterwegs ist, wobei die Karte und die darauf vermerkten Datumsangaben der ersten Fassung des Romans dieses Verborgene zumindest ahnen lassen, während seine Aktivitäten in der zweiten Fassung nur noch in Gestalt nachgelieferter Geschichten rekonstruierbar werden. Die dabei hervorgebrachten Vorgeschichten präsentieren Dinge, die man los sein will, von denen man sich aber erst lösen kann, nachdem sie im Erzählen erinnert wurden. Vorgeschichten zeugen mithin von der Persistenz und Rekurrenz vergangener Verhältnisse.

Was aber folgt nun aus der späten Vergegenwärtigung dieser Vorgeschichten? Während Wilhelm mit seinem Arztberuf die ideelle Nachfolge seines Vaters antritt, liebt Lenardo die »uranfänglichen Zustände« (FA 10, 407). Deshalb möchte er nicht das Erbe des Vaters antreten, sondern will in Amerika neu anfangen. Dem Umstand, dass er dort keineswegs ganz von vorne beginnen, sondern auf die Ländereien seines eigenen Großvaters zurückkehren wird, erklärt der Roman als Rekurs der Enkel auf die Position ihrer Großväter zur Regel. Wilhelm und Lenardo hatten im ersten Buch ein Anwesen passiert, das vom Sohn und Erben verschlossen und für die nachfolgende Generation verwahrt worden war: »damit irgend ein Enkel, in die Neigung und Besitzung des Großvaters eingreifend, alles finde wie dieser es verlassen hat « (FA 1, 406-407). Oder noch einmal pointierter aus dem Mund des Antiquars: »Gewöhnlich zerstreut der Sohn was der Vater gesammelt hat, sammelt etwas anders, oder auf andere Weise. Kann man jedoch den Enkel, die neue Generation abwarten, so kommen dieselben Neigungen, dieselben Ansichten wieder zum Vorschein.« (FA 10, 411) Die schon in den Wahlverwandtschaften beklagte Abkehr der Söhne von den Projekten ihrer Väter, die mit dem Moment beschleunigten gesellschaftlichen Wandels assoziiert wird, biegen die Wanderjahre in die Zyklik eines in aller

\footnotetext{
21 Günter Sasse spricht sowohl mit Bezug auf die Geschichte von Susanne als auch auf die Erinnerung an den Fischerknaben von traumatischen Ereignissen, ohne diese Begriffswahl genauer zu reflektieren. So ist die Rede von »Wilhelms Pubertätstrauma als Motivation seiner Berufswahl« (Sasse [Anm. 8], 113), vom Tod des schönen Jünglings als »das traumatische Erlebnis« (Sasse [Anm. 8], 122). Zwar will er »Lenardos traumatisches Erlebnis mit dem >nußbraunen Mädchen«« (Sasse [Anm. 8], 159) analysieren und hält fest: »Die Geschichte, die Lenardo Wilhelm erzählt, handelt von Adelsprivilegien, unterlassener Hilfeleistung und traumatischer Fixierung. « (Sasse [Anm. 8], 163). Im Rahmen einer Argumentation, die sich vorranging für die sozialen und ökonomischen Um- und Aufbrüche in die Moderne interessiert, gerät die Traumastruktur allerdings nicht genau in den Blick.
} 
Ruhe abzuwartenden Rückgriffs der Enkel auf ihre Großväter zurück. Die Form der narrativen Schleifenbildung, die den Roman formal prägt, hat in der zyklischen Wiederkehr ihre geschichtstheoretische Formel.

Von hier aus lässt sich ein erster Schluss ziehen. Die für die Poetik des Epos prägende Möglichkeit des zurückschreitenden Erzählens ist in den Wanderjahren so gewendet, dass der Ausstieg aus den für den Roman des 18. Jahrhunderts verbindlich gewordenen teleologischen Erzählmustern erprobt werden kann. ${ }^{22}$ Anders als die Lehrjahre, deren Erzählprogramm auf Entwicklung und Veränderung, sei es als Bildung, sei es als Desillusionierung, bedacht ist, sind die Wanderjahre nicht chronologisch voranschreitend erzählt. Stattdessen zielen sie insofern rückwärts, als ihr Sinn in der Rekonstruktion verdeckter Geschichten besteht. Die analeptische Erzählform hat zugleich den Effekt, die Zeitwahrnehmung zu irritieren. In dem Maße, in dem Zeitangaben und Datierungen unterdrückt sind, wird die Zeitenfolge der erzählten Ereignisse unklar. Dies zielt aber nicht unbedingt auf die Prägnanz eines Augenblicks, der nicht vergehen soll. ${ }^{23}$ Vielmehr lässt sich die Aufhebung der Chronologie auch als Einspruch gegen ein geschichtsphilosophisches Modell lesen, das Geschichtszeit als stets kontinuierlich fortschreitende Zeit denkt. Die Vorgeschichten, die Unabgegoltenes und Unerledigtes vergegenwärtigen, durchkreuzen dieses Kontinuum, indem sie sich nicht nach der Reihe, sondern sprunghaft wieder präsentieren. Auf diese Weise machen die Wanderjahre in ihren Stagnationen und Wiederholungen die Wiederkehr zurückliegender Ereignisse wahrnehmbar. Mit diesem Interesse an der untergründigen Persistenz des Vergangenen, dies soll zuletzt zumindest in Ansätzen skizziert werden, korrespondiert die Zeit des Romans mit dem Konzept einer geologischen Tiefenzeit.

\section{IV.}

In seinen bereits in den 1780er Jahren betriebenen und gerade im Entstehungskontext der Wanderjahre intensiv verfolgten mineralogischen und geognostischen Interessen gelangt Goethe zu bemerkenswerten Vorstellungen von einer Zeit der Natur. In einem kurzen Schema über »Chemische Kräfte« notiert er:

\footnotetext{
22 Zur Krise des teleologischen Erzählens in der zweiten Hälfte des 19. Jahrhunderts vgl. Philip Ajouri, Erzählen nach Darwin. Die Krise der Teleologie im literarischen Realismus: Friedrich Theodor Vischer und Gottfried Keller, Berlin, New York 2007.

23 Grundlegend zu Goethes Konzept des erfüllten Augenblicks: Andreas Anglet, Der >ewige Augenblick. Studien zu Struktur und Funktion eines Denkbildes bei Goethe, Köln, Weimar, Wien 1991. Die Thesen Andreas Anglets aufgreifend, zum Teil aber einschränkend und präzisierend, hat Andreas Kolle die Evokation prägnanter Augenblicke in vier Schlüsselepisoden der Wanderjahre analysiert und kommt zu dem Ergebnis, dass die im Frühwerk greifbare Augenblicksemphase in Goethes spätem Roman ambiguiert werde und zwar dergestalt, »dass der Augenblick jedes Mal nicht mehr eindeutig in Richtung einer Ewigkeitsqualität des Moments zeigt, sondern eine bedrohlich-flüchtige Signatur trägt.« So resümiert Kolle: »Die Wanderjahre zeigen: Der flüchtige Augenblick ist schmerzhaft, und die schöne Lösung der Kunst bleibt als poetisches Wunschbild erkennbar.« Andreas Kolle, Instabile Weltverhältnisse. Augenblick und Ambivalenz der Zeitwahrnehmung bei Goethe, Paderborn 2016, 270.
} 
»Die Epochen wo die selbige Gebirgsart erscheint sind nicht gleichzeitig.

Sie hängen von örtlicher Bestimmung ab.

In dem tiefen Meer fand weniger Gebirgsbildung statt.

Fertiges Gebirg zog werdendes an sich aus der flüssigen Masse.

Nachgenannte Epochen folgten sich waren aber auch gleichzeitig.

Schoben sich an einander her.

So ist sehr möglich, daß Granit mehrmals vorkommt.

Ebenso Porphyr, Kalk und alle Formationen.

So ist die Trappformation nur Eine aber nach und nach unter immer wiederkehrenden Bedingungen möglich «(FA 25, 567).

Hier artikuliert sich nicht nur die Einsicht in die nach menschlichem Maßstab intuitiv nicht erfassbare Dauer einer geologischen Tiefenzeit, die Stephen Gould als die von Freud übersehene vierte Kränkung des Menschen neben Kopernikus, Darwin und Freud selbst bezeichnet hat. ${ }^{24}$ In Goethes stichpunktartig festgehaltenen Überlegungen zum Granit als dem vermeintlich ältesten Urgestein schlägt sich auch dasjenige nieder, was der Redaktor der ersten Fassung der Wanderjahre als »ungewisse Zeitrechnung « (FA 10, 162) des Romans bezeichnet. Denn für die Geologie als Erdgeschichte bedeutet der von Goethe notierte Befund, dass der Geognostiker auf Gesteinsschichtungen stößt, die keineswegs eindeutigen Aufschluss über den Ablauf der Erdgeschichte geben. Der Granit führt den Geognostiker nicht an einen ersten Anfang, sondern in ein ungewisses Dunkel: »das Vorhergehende, was den Kern der Erde bildet, ist uns unbekannt «. Goethe muss deshalb vermuten, dass auch »dieses Urgranitgebirge doch auch nur ein Übergang « ist. ${ }^{25}$ Vor diesem Hintergrund erschließt sich, warum Goethes frühe Versuche, aus der unregelmäßigen Schichtung der Gesteine eine Geschichte der Erde zu rekonstruieren, als ein der literarischen Fiktion verwandtes Unternehmen betrieben werden. ${ }^{26}$

Dieses Problem differenziert sich in Goethes späten geologischen Studien weiter aus. Wie Jason Groves gezeigt hat, spielt hier das Interesse an dislozierten Granitblöcken, sogenannten Findlingen, eine wichtige Rolle. Goethe spricht in Bezug auf diese wandernden, von ihrem ursprünglichen Ort entfernten Granitbruchstücke auch

\footnotetext{
24 Stephen J. Gould, Die Entdeckung der Tiefenzeit. Zeitpfeil oder Zeitzyklus in der Geschichte unserer Erde, München 1990. Grundlegend zu Goethes geologischen Studien im Kontext einer Verzeitlichung der Natur vgl. Uwe Pörksen, »Raumzeit. Goethes Zeitbegriff aufgrund seiner sprachlichen Darstellung geologischer Ideen und ihrer Visualisierung «, in: Peter Matussek (Hrsg.), Goethe und die Verzeitlichung der Natur, München 1998, 101-127. An diese Entdeckung der Tiefenzeit mit ihren langen und langsamen Verläufen haben Überlegungen zu Geologie und Literatur insbesondere mit Bezug auf Stifters Erzählen höchst produktiv angeknüpft; Sabine Schneider, »Kulturerosionen. Stifters prekäre geologische Übertragungen«, in: Michael Gamper, Karl Wagner (Hrsg.), Figuren der Übertragung. Adalbert Stifter und das Wissen seiner Zeit, Zürich 2009, 249-269.

25 Goethe, »Bildung der Erde«, FA 25, 527-539, hier: 538.

26 Mit den unterschiedlichen Strategien, die unbekannten Anfänge der Erde zu imaginieren, zu visualisieren und zu erzählen, hat sich Peter Schnyder intensiv befasst. Mit Bezug auf Goethes frühe Texte zum Granit vgl. insbes. Peter Schnyder, »Grund-Fragen. Goethes Text >Über den Granit< als >Ur-ei< der Wissensrepräsentation«, in: Barbara Naumann, Margrit Wyder (Hrsg), »Ein Unendliches in Bewegung «. Das Ensemble der Künste im Wechselspiel mit der Literatur bei Goethe, Bielefeld 2012, 245-263.
} 
als »Granitgeschiebe «. ${ }^{27}$ Vorgänge des Schiebens und Verschiebens dienen dem Neptunisten Goethe, dem die vulkanische Vorstellung eines »Hebens und Drängens «, »Aufwälzens und Quetschens«, »Schleuderns und Schmeißens« eher »widerwärtig von jeher erscheinen mußte « (FA 25, 649), als grundlegende Modelle erdgeschichtlicher Prozesse. ${ }^{28} \mathrm{Ob}$ dieses Geschiebe des Granit durch die Fluten eines Urozeans oder, wie einige Zeitgenossen bereits vorschlagen, durch die Eisbildung einer vorübergehenden Kaltzeit zuwege gebracht worden ist, lässt Goethe offen. Entscheidend ist aber, dass hier eine Dynamik angenommen wird, die auch gegenwärtig und zukünftig noch für kontingente Veränderungen der Erdoberfläche sorgt. Mit der Ungleichzeitigkeit des Gleichförmigen und der Gleichzeitigkeit von Ungleichem, dem Prinzip der Wiederholung, der Uneinsehbarkeit eines absoluten Anfangs und der permanenten Dynamik des Verschiebens liegen in Goethes Geologie ebenjene Denkfiguren bereit, die sich auch für die narrative Zeitorganisation der Wanderjahre als prägend herausgestellt haben: allen voran die Verschiebung einzelner Episoden und die daraus resultierende Umstellung der zeitlichen Chronologie, aber auch die Phänomene der Wiederkehr und des entzogenen Anfangs. Goethe scheint in der narrativen Gestaltung der Wanderjahre Modelle einer Zeit der Natur durchgespielt zu haben.

Dieser Deutung kommt entgegen, dass die Geologie im Allgemeinen und der Granit im Besonderen im Roman einen unübersehbaren Motivkomplex bilden. Bereits in den ersten Gesprächen zwischen Wilhelm, Felix und Jarno-Montan, der sich autodidaktisch zum Bergbauexperten gemacht hat, bildet die Bestimmung von Gesteinssorten sowie die daran geknüpfte Frage nach dem Ursprung und dem Alter der Welt einen zentralen Gesprächsgegenstand. Die Debatten um konkurrierende Modelle der Erdentstehung werden im neunten Kapitel des zweiten Buchs auf dem Bergfest noch einmal aufgegriffen. ${ }^{29}$ Die Thematik der Erdgeschichte verdichtet sich schließlich in einem metapoetischen Objekt. Auf ihrem Abstieg vom Gebirge klettert Wilhelms Sohn Felix in eine »Kluft des schwarzen Gesteines« (FA 10, 302). Goethes Beschreibungen von Granitgebirgen fixieren sich immer wieder auf die Entstehung von vertikalen, die horizontalen Schichtungen durchschneidenden Klüfte, seien es Gangklüfte oder Flözklüfte, durch die bestehende horizontale Schichtungen in rhombenförmige Blöcke geteilt werden. Aus einer solchen Kluft als Index späterer Durchstöße durch zuvor gebildete Schichtungen bringt Felix »ein Kästchen mit, nicht größer als ein kleiner Oktavband«, das vom Erzähler auch als »Pracht-

\footnotetext{
27 Goethe, »Granitarbeiten in Berlin«, FA 25, 639-640, hier: 639. Jason Groves verfolgt hier hauptsächlich Goethes Ansätze zu einer Theorie der Eiszeiten, in der sich der Granit als Index möglicher klimatischer Veränderungen herausstellt. Groves macht diese Überlegungen aber nur am Rand für die Form der Wanderjahre produktiv. Jason Groves, »Goethe's petrofiction. Reading the >Wanderjahre< in the Anthropocene«, Goethe yearbook 22 (2015), 95-113.

28 So Goethe in seiner Rezension des Buchs »Über die Gestalt und die Urgeschichte der Erde von K.F. Klöden $1829 \ll$.

$29 \mathrm{Zu}$ Goethes Positionierung im Streit um Neptunismus und Vulkanismus vgl. Michaela Haberkorn, $\mathrm{Na}$ turhistoriker und Zeitenseher. Geologie und Poesie um 1800. Der Kreis um Abraham Gottlob Werner, Frankfurt a. M., Bern, Berlin 2004, 65-70. Allgemein zu einer Einschätzung Goethes geologischer Schriften und der Motive des Gesteins und des Bergbaus in seinen literarischen Texten, vgl. Wolf von Engelhardt, Goethe im Gespräch mit der Erde. Landschaft, Gesteine, Mineralien und Erdgeschichte in seinem Leben und Werk, Köln, Weimar, Wien 2003; zu den Wanderjahren: 256-259.
} 
bändchen « (FA 10, 302) bezeichnet wird. Mit diesem Kistchen, dessen Fundort ein geologisches Relikt, dessen äußere Erscheinung aber ein Buch evoziert, ${ }^{30}$ bietet der Roman sehr deutlich an, die Erdgeschichte als Modell der im Roman erprobten Anordnung von Geschichten zu lesen.

Für diesen Zusammenhang trägt der Schluss des Romans, in dem das Kästchen zu einem zentralen Handlungsrequisit wird, einen letzten Anhaltspunkt bei. Felix und Wilhelm hatten auf dem nahegelegenen Gut des Oheims Hersilie kennengelernt, bei der sie das mitgebrachte Kästchen lassen. Im dritten Buch deutet sich, wenn auch schwach, eine Dreiecksbeziehung an: Während sich Felix in die ältere Hersilie verliebt, wendet sich Hersilie durchaus generationsgerecht eher Wilhelm zu. In dieser Verwicklung spielt das Kästchen eine entscheidende Rolle, findet doch ausgerechnet Hersilie am Ende des Romans den dazu passenden Schlüssel, den sie abzeichnet und als Skizze an Wilhelm schickt:

»Hier aber, mein Freund, nun schließlich zu dieser Abbildung des Rätsels was sagen Sie? Erinnert es nicht an Pfeile und Widerhaken? Gott sei uns gnädig! Aber das Kästchen muß zwischen mir und Ihnen erst uneröffnet stehen, und dann eröffnet das Weitere befehlen. Ich wollte, es fände sich gar nichts drinnen und was ich sonst noch wollte und was ich sonst noch alles erzählen könnte - doch sei Ihnen das vorenthalten, damit Sie desto eiliger sich auf den Weg machen.«(FA 10, 599)

In der Forschung werden Kästchen und Schlüssel meist als Hinweis auf die Grenzen der Naturerkenntnis oder auf die Grenzen der Hermeneutik gedeutet: Weder lässt sich das Geheimnis der Natur erschließen, noch lässt sich der Roman restlos aufschlüsseln. ${ }^{31}$ Diesen Verrätselungscharakter betont die Szene zwar, sie koppelt ihn aber auch an eine für den Roman charakteristische Zeitregie. Hersilie erzählt nicht sofort alles, was sie zu dem gewählten Zeitpunkt erzählen könnte, sondern will Wilhelm als dem Leser des Briefes etwas durchaus Berichtbares vorenthalten. Ihre Mitteilung, die hier auch auf die Chronologie des Romans durchschlägt, folgt mithin dem Gesetz des temporalen Aufschubs. Noch dazu liefert Hersilie in Gestalt einer Nachschrift eine Überlegung nach, die einen wiederkehrenden Begriff der Geologie Goethes aufgreift: »Und was das wieder vor Umstände sind! Das schiebt sich und verschiebt sich« (FA 10, 599). Nimmt man die Technik des Schiebens und Ver-

\footnotetext{
30 Auf die Annäherung von Erdgeschichte und Kulturgeschichte in der Orientierung der Geologie an der Archäologie und der Rede von den Denkmälern der Erdgeschichte hat Peter Schnyder hingewiesen in dem Aufsatz »übereinander/nacheinander. Zur Metaphorologie der Schicht«, in: Gunhild Berg, Martina King, Reto Rössler (Hrsg.), Metaphorologien der Exploration und Dynamik 1800/1900. Historische Wissenschaftsmetaphern und die Möglichkeiten ihrer Historiographie, Hamburg 2018, 84-99.

31 Larrett bezieht die romaninternen Diskussionen des Schlüssels auf Goethes eigene Rede vom Wilhelm Meister-Komplex als einem Werk, das nicht restlos aufgeht (>inkalkulabel<). William Larrett, »>Weder Kern noch Schaleく. The >Novel< epistemology of Goethe's >Wilhelm Meisters Wanderjahre« «, Publications of the English Goethe Society 56 (1985/86), 38-55, hier: 39-41. Im Hinweis auf alchemistischhermetisches Wissen des 17. Jahrhunderts hat Diethelm Brüggemann das verschlossene, kurz geöffnete aber womöglich leere Kästchen als Variation auf das leere Schachtelbuch aus der Via Lucis des Comenius gedeutet, mithin als Allegorie auf ein Geheimwissen von der Natur, das sich paradoxerweise nur dem ohnehin schon wissenden Weisen erschließt. Diethelm Brüggemann, Makarie und Mercurius. Goethes Wilhelm Meisters Wanderjahre als hermetischer Roman, Frankfurt a. M., Bern, Berlin 1999, 85.
} 
schiebens auch als Gesetz der achronologischen Erzählung ernst, dann reagiert der Roman nicht nur auf das Problem, Lebensgeschichten als ordentlich gefügte Ketten von Ursachen und Folgen zu erzählen. Der in der Romanform erfahrbare Entzug der Chronologie verweist zugleich auf die ungleich größeren Herausforderungen einer ungewissen erdgeschichtlichen Zeitrechnung. So gelesen zieht das rückwärtsschreitende Erzählen der Wanderjahre die formalen Konsequenzen aus der Einsicht, dass jedes Erzählen ein Erzählen medias in res ist, in dessen komplexem Gefüge sich jeder Anfang als Moment des Übergangs erweist.

Funding Open Access funding provided by Projekt DEAL.

Open Access Dieser Artikel wird unter der Creative Commons Namensnennung 4.0 International Lizenz veröffentlicht, welche die Nutzung, Vervielfältigung, Bearbeitung, Verbreitung und Wiedergabe in jeglichem Medium und Format erlaubt, sofern Sie den/die ursprünglichen Autor(en) und die Quelle ordnungsgemäß nennen, einen Link zur Creative Commons Lizenz beifügen und angeben, ob Änderungen vorgenommen wurden.

Die in diesem Artikel enthaltenen Bilder und sonstiges Drittmaterial unterliegen ebenfalls der genannten Creative Commons Lizenz, sofern sich aus der Abbildungslegende nichts anderes ergibt. Sofern das betreffende Material nicht unter der genannten Creative Commons Lizenz steht und die betreffende Handlung nicht nach gesetzlichen Vorschriften erlaubt ist, ist für die oben aufgeführten Weiterverwendungen des Materials die Einwilligung des jeweiligen Rechteinhabers einzuholen.

Weitere Details zur Lizenz entnehmen Sie bitte der Lizenzinformation auf http://creativecommons.org/ licenses/by/4.0/deed.de. 